\title{
Synchronizing the simplest classical system and then quantizing it
}

\author{
Paweł Kurzyński ${ }^{*}$ \\ Faculty of Physics, A. Mickiewicz University, Uniwersytetu Poznańskiego 2, 61-614 Poznań, Poland \\ and Centre for Quantum Technologies, National University of Singapore, 3 Science Drive 2, 117543 Singapore, Singapore
}

(Received 8 March 2020; accepted 20 July 2020; published 24 August 2020)

\begin{abstract}
We propose a discrete synchronization model of a finite $d$-level system and discuss what happens once superposition of states is allowed. The model exhibits various asymptotic behaviors that depend on the initial state. In particular, two antagonistic phenomena can occur: a transition into a product of two basis states and an entanglement generation. Next, we generalize this model and show that it is possible to phase lock a periodic dynamics of a single qubit to a periodic dynamics of a single qudit. Finally, we present a spin system whose dynamics is a continuous-time analog of the above discrete model.
\end{abstract}

DOI: 10.1103/PhysRevResearch.2.033289

\section{INTRODUCTION}

Synchronization is a process in which two or more interacting oscillators adjust their rhythms. It can be found in many areas of classical physics [1] and was also studied in the quantum regime [2-4]. Recently, an interesting question was asked [5]: What is the smallest quantum system that can be synchronized? The authors of [5] focused on a single spin-s system and argued that synchronization is possible for $s=1$ (qutrit), but not for $s=1 / 2$ (qubit). This work stimulated further research [5-8] and, at the moment, there are arguments for [8] and against [5] single spin-1/2 synchronization. These contrary arguments originate from a problem of how to define a limit cycle in quantum dynamics. Limit cycles are assumed to be prerequisites for synchronization [9], but they can only emerge in nonlinear systems [10]. However, quantum dynamics is fundamentally linear (apart from the measurement process), and therefore it is crucial to gain a deeper understanding of how synchronization works in the quantum domain.

Here, we propose an alternative approach to spin- $s$ synchronization which avoids the limit cycle problem. Let us start with the following argument. Consider a spin precessing in a magnetic field, a model studied in Refs. [5-8]. The system is described by two quantum numbers: $s$ (total spin) and $s_{n}$ (spin projection onto the $\mathbf{n}$ axis). Let the precession be about the $\mathbf{m}$ axis that is orthogonal to $\mathbf{n}$. It is well known that in this case, one can observe periodic oscillations of $s_{n}$. There are two possible ways in which these oscillations can cease: either (i) the spin state becomes a mixture of eigenstates of $\hat{S}_{\mathbf{m}}$ or (ii) the total spin decays to $s=0$. The crucial observation

\footnotetext{
*pawel.kurzynski@amu.edu.pl

Published by the American Physical Society under the terms of the Creative Commons Attribution 4.0 International license. Further distribution of this work must maintain attribution to the author(s) and the published article's title, journal citation, and DOI.
}

is that in case (i), the amplitude of oscillations is not lost since $\left\langle S_{\mathbf{n}}^{2}\right\rangle>0$. One rather loses information about the value of $s_{n}$. A classical analog would correspond to losing information about the phase of oscillations-the oscillations still occur, one just does not know their exact state. Only in case (ii) is the amplitude of oscillations truly lost. However, if one considers an elementary spin system, the $s$ number is fixed. Unlike in the case of composite systems, such as two spin-1/2 particles, an elementary spin cannot decrease its total spin value to zero.

Under the above argument, an oscillating elementary spin system does not suffer true amplitude losses. The oscillations are fully described by the phase. This suggests a parallel to the classical Poincare phase oscillator (PPO) $\dot{\theta}=\omega$, where $\theta \in[0,2 \pi)$ is the phase and $\omega$ is the frequency [11]. The PPO can be visualized as a particle moving on a ring. The particle cannot go beyond its circular track, and hence there is no need for a limit cycle. Any dissipation can only disturb the phase, but cannot cease the motion. To justify our approach, let us stress that many synchronization models, such as the Kuramoto model [12], are based on PPOs. Moreover, the PPO simplification allows one to focus on a more fundamental dissipative aspect of synchronization-phase locking.

The goal of this work is to use the above PPO analogy to derive a quantum synchronization model of an arbitrary spin- $s$ system or, more generally, a qudit. Our guiding idea comes from the following two questions: What is the smallest classical system that can be synchronized? How can this system be quantized? What is meant by the smallest is ambiguous. We choose an information-theoretic point of view-the smallest system is the one whose encoding requires a minimal amount of information. Therefore, we focus on $d$-level systems encoded on $\log _{2} d$ classical bits. Our main motivation behind this approach comes from the fact that qudits are discrete-state systems. In addition, both a classical $d$-level system and a qudit can store the same amount of classical information. This fact allows one to compare the two systems from the computational efficiency perspective, especially in the context of any possible quantum information-processing advantage. Moreover, our approach is along the lines of research on 
complex dynamics of finite-state systems and pseudochaos [13-15]. This line of research is particularly important since realistic computers, both classical and quantum, are fundamentally finite-state machines that cannot support the continuous nature of chaotic systems [15].

First, we are going to discretize the PPO synchronization model. Next, we quantize it and focus on dynamics in which superposed initial states are allowed. The proposed model offers an insight into a phase-locking mechanism and, more importantly, on how this mechanism works and what it offers once one goes quantum. In particular, we are going to show that depending on an initial state of the oscillator and the stimulus, the phase locking can lead to two antagonistic processes: a transition to a product of two basis states and an entanglement generation. Then, we are going to show that it is possible to phase lock a single qubit dynamics to a single qudit dynamics. Notice that the proposed quantum model is discrete in both state space and time. Therefore, it can be formulated in terms of quantum gates and maps that are sequentially applied to the system's state, just like any other quantum information-processing protocol. Nevertheless, at the end, we are going to show that there is some parallel between this model and an open continuous-time spin dynamics.

\section{PPO SYNCHRONIZATION MODEL}

Let us recall a simple continuous model of synchronization (see, e.g., [10]). Consider a stimulus whose frequency is $\Omega$ and whose phase variable is $\theta \in[0,2 \pi)$. Its free evolution is given by

$$
\dot{\theta}=\Omega \text {. }
$$

Next, consider an oscillator whose frequency is $\omega$ and whose phase variable is $\varphi \in[0,2 \pi)$. The evolution of this oscillator is affected by the stimulus in the following way:

$$
\dot{\varphi}=\omega+K f(\theta-\varphi),
$$

where $f(\theta-\varphi)$ is a periodic function that describes the way the oscillator responds to the stimulus and $K$ measures the strength of this response. One often takes $f(\theta-\varphi)=\sin (\theta-$ $\varphi$ ), which implies the following behavior: the oscillator speeds up if $\varphi$ is behind $\theta$, and slows down if $\varphi$ is ahead of $\theta$. It is useful to define the phase difference $\Delta=\theta-\varphi$, whose evolution is given by

$$
\dot{\Delta}=\Gamma-K f(\Delta),
$$

where $\Gamma=\Omega-\omega$. The phenomenon of phase locking occurs once the phase difference settles down to some constant value $\Delta^{*}$ (here, $*$ denotes a fixed point, not a complex conjugation), which implies $\Gamma=K f\left(\Delta^{*}\right)$. This equation can be satisfied if $\Gamma / K$ is in the image of $f(\Delta)$, which defines the range of entrainment. If $\Gamma / K$ is outside of this range, the oscillator is not able to adjust its rhythm to the stimulus.

\section{DISCRETE SYNCHRONIZATION MODEL}

In this section, we are going to discretize Eqs. (1)-(3). At this point, some readers may think of the circle map

$$
\alpha_{t+1}=\alpha_{t}+\Gamma-\frac{K}{2 \pi} \sin \left(2 \pi \alpha_{t}\right)
$$

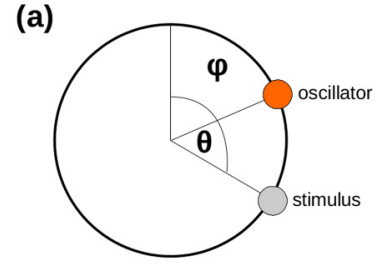

(c)

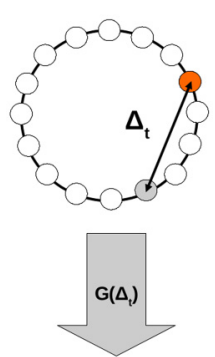

(b)
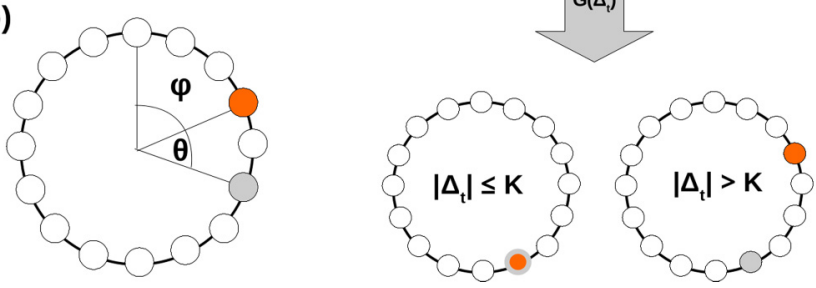

FIG. 1. Discretization of the PPO synchronization model: (a) two continuous PPOs; (b) two discrete PPOs; (c) the action of the map $G\left(\Delta_{t}\right)$-if the stimulus is within the distance $K$ from the oscillator, the oscillator jumps to the stimulus' position, but when this distance is larger than $K$, the oscillator does not move.

which is a particular time-discrete version of (3). However, the circle map is not what we are looking for since the variable $\alpha_{t}$ that describes the state of the system is continuous. The goal is to further discretize the circle map, i.e., to discretize the set of the system's states.

Let us assume that the phases of the stimulus and the oscillator are discrete and can take $d$ different values $\theta_{t}, \varphi_{t} \in$ $S=\{0,1,2,3, \ldots, d-1\}$. The equations (1)-(3) become

$$
\begin{aligned}
\theta_{t+1} & =\theta_{t}+\Omega, \\
\varphi_{t+1} & =\varphi_{t}+\omega+G\left(\theta_{t}-\varphi_{t}\right), \\
\Delta_{t+1} & =\Delta_{t}+\Gamma-G\left(\Delta_{t}\right),
\end{aligned}
$$

where $G\left(\theta_{t}-\varphi_{t}\right)$ is an analog of $K f\left(\theta_{t}-\varphi_{t}\right)$ and we assume that all values are taken mod $d$. Note,that $\theta_{t}, \varphi_{t} \in S$ for all $t$, which implies that $\Omega$ and $\omega+G\left(\theta_{t}-\varphi_{t}\right)$ must be in $S$ too. Since the choice of $\omega$ should not depend on $G\left(\theta_{t}-\varphi_{t}\right)$, and vice versa, we assume that both of them are integers from $S$.

Phase locking implies that after $\tau$ steps, the phase difference $\Delta_{t}$ is fixed, i.e., $\Delta_{t}=\Delta^{*}$ for $t \geqslant \tau$. This implies $G\left(\Delta^{*}\right)=\Gamma$, and hence a prerequisite for phase locking is that $\Gamma$ is one of the outcomes of $G\left(\Delta_{t}\right)$. Let us choose

$$
G_{K}\left(\Delta_{t}\right)=\left\{\begin{array}{c}
\Delta_{t} \text { if } \Delta_{t} \in E_{K}, \\
0 \text { else }
\end{array}\right.
$$

where $E_{K}=\{0, \ldots, K, d-K, \ldots, d-1\}$ and $K \leqslant\left\lfloor\frac{d}{2}\right\rfloor$. Notice that since $-\Delta_{t} \equiv d-\Delta_{t} \bmod d$, the condition $\Delta_{t} \in E_{K}$ can be rewritten as $\left|\Delta_{t}\right| \leqslant K$. Moreover, if $K=\left\lfloor\frac{d}{2}\right\rfloor$, then $E_{K}=S$ and the oscillator always responds to the stimulus, no matter the system's state. An intuitive explanation of how $G_{K}\left(\Delta_{t}\right)$ works is presented in Fig. 1.

In Appendix A, we prove that phase locking occurs iff $\Gamma \in E_{K}$. This happens after time $\tau$ and, for all later times the evolution of the oscillator is given by

$$
\varphi_{\tau+t}=\theta_{\tau+t}-\Gamma .
$$

An example of such dynamics is presented in Fig. 2. If $\Gamma \notin$ $E_{K}$, then there is a phase drift- $\Delta_{t}$ constantly changes. In this 


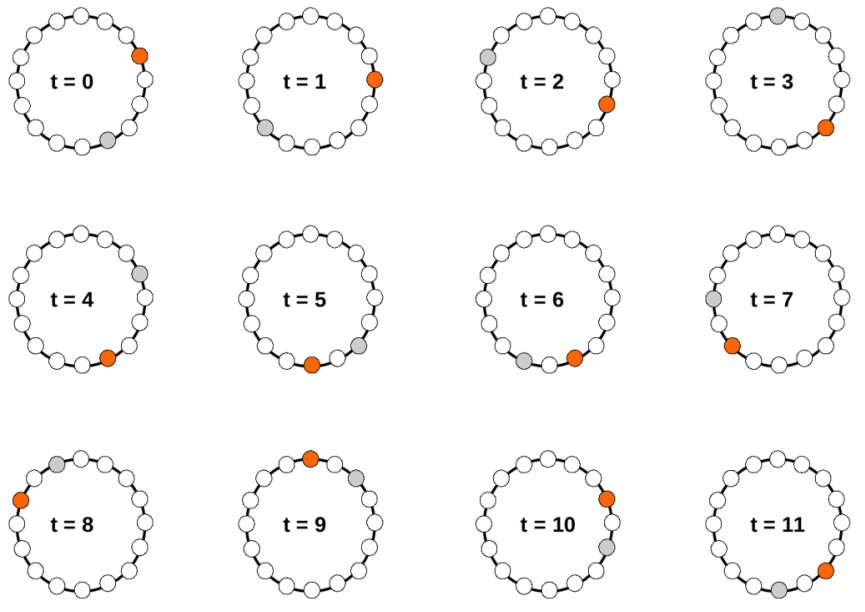

FIG. 2. An example evolution of the model described by Eqs. (5)-(7). The stimulus and the oscillator correspond to the gray and orange dots, respectively. The parameters of the model are $\omega=$ $1, \Omega=3$, and $K=2$. The phase locking occurs at $t=6$. For $t \geqslant 6$, the stimulus is always $\Gamma=\Omega-\omega=2$ steps ahead of the oscillator.

case, $\Delta_{t}$ changes periodically due to the finiteness of the state space.

At this point, it is clear that the smallest classical system that can be synchronized is a single bit. This is because $d=2$ is the smallest value for which the above dynamics is not trivial. Still, note that in this case, there are only two possible values of $\Omega, \omega$, and $K$ : either zero or one. Therefore, the corresponding two-bit dynamics is relatively simple. We will come back to this issue later.

\section{QUANTUM SYNCHRONIZATION MODEL}

In this section, we are going to quantize the above system. Let us first reformulate the model in a vector space formalism. Instead of writing $\theta_{t}$ and $\varphi_{t}$, we will write $\left|\psi_{t}\right\rangle=\left|\theta_{t}\right\rangle \otimes\left|\varphi_{t}\right\rangle \in$ $\mathbb{C}^{d} \otimes \mathbb{C}^{d}$. Let us define an orthonormal basis $\{|i\rangle\}_{i=0}^{d-1}$ and let us assume that both $\left|\theta_{t}\right\rangle$ and $\left|\varphi_{t}\right\rangle$ are some vectors from this basis. Let us also define two Hermitian operators,

$$
\hat{\theta}=\left(\sum_{i=0}^{d-1} i|i\rangle\langle i|\right) \otimes \hat{\mathbb{1}}, \quad \hat{\varphi}=\hat{\mathbb{1}} \otimes\left(\sum_{i=0}^{d-1} i|i\rangle\langle i|\right) .
$$

These operators allow one to recover $\theta_{t}=\left\langle\psi_{t}|\hat{\theta}| \psi_{t}\right\rangle$ and $\varphi_{t}=$ $\left\langle\psi_{t}|\hat{\varphi}| \psi_{t}\right\rangle$.

Next, let us define unitary operators $\hat{U}_{\Omega}$ and $\hat{U}_{\omega}$,

$$
\hat{U}_{\Omega}\left|\theta_{t}\right\rangle=\left|\theta_{t}+\Omega\right\rangle, \quad \hat{U}_{\omega}\left|\varphi_{t}\right\rangle=\left|\varphi_{t}+\omega\right\rangle,
$$

where the mod $d$ convention is used. They describe the free evolution during which the stimulus and the oscillator follow their own rhythms. Eventually, let us define an operator $\hat{G}_{K}$ that acts on both systems,

$$
\hat{G}_{K}\left|\theta_{t}\right\rangle \otimes\left|\varphi_{t}\right\rangle=\left\{\begin{array}{l}
\left|\theta_{t}\right\rangle \otimes\left|\theta_{t}\right\rangle \text { if } \Delta_{t} \in E_{K} \\
\left|\theta_{t}\right\rangle \otimes\left|\varphi_{t}\right\rangle \text { else }
\end{array}\right.
$$

whose action mimics the one of (8). Note that $\hat{G}_{K}$ is not unitary; however, together with $\hat{U}_{\Omega}$ and $\hat{U}_{\omega}$, it allows one to iterate the state of the system in the following way:

$$
\left|\psi_{t+1}\right\rangle=\left(\hat{U}_{\Omega} \otimes \hat{U}_{\omega}\right) \hat{G}_{K}\left|\psi_{t}\right\rangle .
$$

The lack of unitarity of $\hat{G}_{K}$ is related to its irreversibility. Note that this operator changes many different inputs into the same output $\left|\theta_{t}\right\rangle \otimes\left|\theta_{t}\right\rangle$. Such a transformation can be realized via Kraus operators. Equivalently, it can be realized unitarily by adding an ancillary system. We are going to follow the second approach.

We assume that the ancillary system has $d+1$ levels with the corresponding basis vectors $\{|\overline{0}\rangle\} \cup\{|i\rangle\}_{i=0}^{d-1}$, where $|\overline{0}\rangle$ is the initial state. Let us introduce a unitary operator $\hat{V}_{K}$ whose action is given by

$$
\hat{V}_{K}\left|\theta_{t}\right\rangle \otimes\left|\varphi_{t}\right\rangle \otimes|\overline{0}\rangle= \begin{cases}\left|\theta_{t}\right\rangle \otimes\left|\theta_{t}\right\rangle \otimes|\overline{0}\rangle & \text { if } \Delta_{t}=0, \\ \left|\theta_{t}\right\rangle \otimes\left|\varphi_{t}\right\rangle \otimes|\overline{0}\rangle & \text { if } \Delta_{t} \notin E_{K}, \\ \left|\theta_{t}\right\rangle \otimes\left|\theta_{t}\right\rangle \otimes\left|\Delta_{t}\right\rangle & \text { else. }\end{cases}
$$

The operator $\hat{V}_{K}$ implements $\hat{G}_{K}$ on the first two systems. Moreover, note that the choice of $\hat{V}_{K}$ is not unique. The reason why we chose it as (14) is explained later. Next, we impose that the ancilla is reset to $|\overline{0}\rangle$ after each application of $\hat{V}_{K}$ so that one can iterate the evolution more than once. This resetting is a source of dissipation that makes phase locking possible. The above leads to the following formulation of Eqs. (5)-(7):

$$
\begin{aligned}
\theta_{t+1} & =\operatorname{Tr}\left\{\rho_{t+1} \hat{\theta}\right\}, \\
\varphi_{t+1} & =\operatorname{Tr}\left\{\rho_{t+1} \hat{\varphi}\right\}, \\
\Delta_{t+1} & =\operatorname{Tr}\left\{\rho_{t+1}(\hat{\theta}-\hat{\varphi})\right\},
\end{aligned}
$$

where

$$
\rho_{t+1}=\operatorname{Tr}_{a n c}\left\{\hat{\mathbb{U}}\left(\rho_{t} \otimes|\overline{0}\rangle\left\langle\left.\overline{0}\right|_{\text {anc }}\right) \hat{\mathbb{U}}^{\dagger}\right\},\right.
$$

and

$$
\hat{\mathbb{U}}=\left(\hat{U}_{\Omega} \otimes \hat{U}_{\omega} \otimes \hat{\mathbb{1}}\right) \hat{V}_{K}
$$

\section{Starting in a superposition of basis states}

Up to now, the system's state was assumed to be a product of two basis vectors, and hence one could choose either Eqs. (5)-(7) or (15)-(17) to describe its dynamics. However, the latter set of equations allows one to consider a much bigger set of initial states. Below, we are going to focus on the case $\Gamma \in E_{K}$ and discuss what happens if the initial state is a superposition of basis vectors.

Let us first consider a case in which the stimulus is prepared in one of the basis states, whereas the oscillator is prepared in a superposition. The initial state is $\rho_{0}=\left|\psi_{0}\right\rangle\left\langle\psi_{0}\right|$, where

$$
\left|\psi_{0}\right\rangle=\left|\theta_{0}\right\rangle \otimes\left(\sum_{i=0}^{d-1} \alpha_{i}|i\rangle\right) .
$$

The asymptotic behavior of the system stems from (9). The phase locking of all terms occurs after $\tau$ steps and, for $t>\tau$, the system's state is described by $\rho_{t}=\left|\psi_{t}\right\rangle\left\langle\psi_{t}\right|$, where $\left|\psi_{t}\right\rangle=$ $\left|\theta_{t}\right\rangle \otimes\left|\theta_{t}-\Gamma\right\rangle$ and $\theta_{t}=\theta_{0}+\Omega t$. This state is a product of two basis vectors. 
Next, we are going to show that a product of the superposed oscillator and the superposed stimulus evolves into an entangled asymptotic state. Interestingly, the entanglement is generated in a dissipative process [16] that depends on a particular choice of $\hat{V}_{K}$ in (14).

Consider $\rho_{0}=\left|\psi_{0}\right\rangle\left\langle\psi_{0}\right|$, where

$$
\left|\psi_{0}\right\rangle=\left(\sum_{i=0}^{d-1} \alpha_{i}|i\rangle\right) \otimes\left(\sum_{j=0}^{d-1} \beta_{j}|j\rangle\right) .
$$

Due to phase locking, the asymptotic state $\rho_{t}$ (for $t>\tau$ ) must be in the subspace $\mathcal{S}_{p l}$ spanned by $\{|i\rangle \otimes|i-\Gamma\rangle\}_{i=0}^{d-1}$ : the phase-locked subspace. The state $\rho_{t}$ is entangled if it has any coherences, i.e., nonzero off-diagonal terms with respect to the basis $\{|i\rangle \otimes|i-\Gamma\rangle\}_{i=0}^{d-1}$. If $\rho_{t}$ has coherences, the partial transposition will move them outside of $\mathcal{S}_{p l}$ and the operator $\rho_{t}^{T_{O}}$ will be of the form $\sigma_{p l} \oplus \bar{\sigma}_{p l}$, where $T_{O}$ denotes partial transposition (transposition in the oscillator subspace). The part $\sigma_{p l}$ is inside $\mathcal{S}_{p l}$, whereas $\bar{\sigma}_{p l}$ is outside of $\mathcal{S}_{p l}$. Moreover, $\bar{\sigma}_{p l}$ is traceless (since there are no diagonal terms outside of $\mathcal{S}_{p l}$ ), Hermitian, and nonzero. This implies that $\rho_{t}^{T_{O}}$ has negative eigenvalues, and thus $\rho_{t}$ is entangled [17,18]. In Appendix B, we show that if (21) contains terms

$$
\left|\Delta_{0}\right\rangle=\alpha_{i} \beta_{i-\Delta_{0}}|i\rangle \otimes\left|i-\Delta_{0}\right\rangle+\alpha_{i^{\prime}} \beta_{i^{\prime}-\Delta_{0}}\left|i^{\prime}\right\rangle \otimes\left|i^{\prime}-\Delta_{0}\right\rangle,
$$

where $\Delta_{0}=0,1, \ldots, d-1$, then $\rho_{t}$ is almost certainly entangled. More precisely, if (21) contains terms $\left|\Delta_{0}\right\rangle$, then there is only a set of measure zero of amplitudes $\alpha_{i}$ and $\beta_{j}$ for which $\rho_{t}$ may not be entangled.

\section{SYNCHRONIZATION OF A SINGLE QUBIT}

The above quantum model works for an arbitrary $d$, just like its classical precursor. In particular, it works for $d=2$, and hence the smallest quantum system that can be synchronized is a single qubit. Still, as in the classical discrete case, the corresponding two-qubit dynamics is relatively simple and the only possible choices of $K$ (one or zero) imply that either the oscillator is slaved to the stimulus or both systems evolve independently. This might be a reason for some researchers to doubt whether the model describes true synchronization [9]. Therefore, in order to show that a single qubit can be synchronized in a more complex way, we are going to generalize the previous model and consider a synchronization of a qubit to a qudit stimulus.

Let us assume that the stimulus is a $d$-level system, $\theta_{t}=$ $0,1, \ldots, d-1$, where $d$ is even, and the oscillator is a qubit, $\varphi_{t}=0,1$. The free dynamics of the stimulus is going to be the same as in the previous case, but, for simplicity, we fix $\left|\theta_{0}\right\rangle=|0\rangle$, and hence $\left|\theta_{t}\right\rangle=\hat{U}_{\Omega}^{t}|0\rangle=|\Omega t\rangle$. Moreover, $\Omega$ is chosen to be a divisor of $d$. For example, if $d=12$, then $\Omega=1,2,3,4,6,12$. Such a choice guarantees that after $d$ steps, the stimulus returns to $\theta_{d}=0$.

The dynamics of the oscillator is going to be based on a unitary transformation,

$$
\begin{gathered}
\hat{R}|0\rangle=\cos \frac{\pi}{d}|0\rangle+\sin \frac{\pi}{d}|1\rangle, \\
\hat{R}|1\rangle=-\sin \frac{\pi}{d}|0\rangle+\cos \frac{\pi}{d}|1\rangle .
\end{gathered}
$$

This transformation can be visualized in the Bloch-sphere picture as a $\frac{2 \pi}{d}$ rotation about the $y$ axis. The oscillator's frequency $\omega$ is also chosen to be a divisor of $d$. The single step of the oscillator's free evolution is generated by $\hat{R}^{\omega}$, which corresponds to a $\frac{2 \pi \omega}{d}$ rotation about the $y$ axis. It is convenient to introduce the following qubit states: $\left|\chi_{k}\right\rangle=\hat{R}^{k}|0\rangle$, where $k=0,1, \ldots, d-1$.

Next, let us introduce a new interaction operator that requires an ancillary qubit (initially prepared in the state $|0\rangle$ ),

$\hat{W}_{K}\left|\theta_{t}\right\rangle \otimes\left|\varphi_{t}\right\rangle \otimes|0\rangle=\left\{\begin{array}{c}\left|\theta_{t}\right\rangle \otimes\left|\varphi_{t}\right\rangle \otimes|0\rangle \text { if }\left|\theta_{t}-\frac{d}{2} \varphi_{t}\right|>K, \\ \left|\theta_{t}\right\rangle \otimes\left|\chi_{\theta_{t}}\right\rangle \otimes|1\rangle \text { else. }\end{array}\right.$

After each application of $\hat{W}_{K}$, the state of the ancilla is reset to $|0\rangle$. In simple words, if $\left|\theta_{t}-\frac{d}{2} \varphi_{t}\right| \leqslant K$, then $\hat{W}_{K}$ measures the oscillator qubit in the $\{|0\rangle,|1\rangle\}$ basis and changes its state to $\left|\chi_{\theta_{t}}\right\rangle$. Let us recall that all values are taken $\bmod d$, as in the previous case. The multiplication of $\varphi_{t}$ by $\frac{d}{2}$ relabels the states of the qubit $\{0,1\} \rightarrow\left\{0, \frac{d}{2}\right\}$, and therefore they can be compared to the states of the stimulus. Intuitively, the dynamics can be described as follows. The stimulus is a particle moving on a discrete ring, as in all the cases considered so far. On the other hand, the oscillator is described by a Bloch vector that rotates in the $x z$ plane. The operator $\hat{W}_{K}$ tries to make the Bloch vector rotate at the particle's pace.

A single step of the evolution is given by

$$
\rho_{t+1}=\operatorname{Tr}_{a n c}\left\{\hat{\mathbb{Q}}\left(\rho_{t} \otimes|0\rangle\left\langle\left. 0\right|_{\text {anc }}\right) \hat{\mathbb{Q}}^{\dagger}\right\},\right.
$$

where

$$
\hat{\mathbb{Q}}=\left(\hat{U}_{\Omega} \otimes \hat{R}^{\omega} \otimes \hat{\mathbb{1}}\right) \hat{W}_{K},
$$

and $\rho_{0}=|0\rangle\langle 0| \otimes \sigma_{0}$ is an initial state of the stimulus and the oscillator. This implies that $\rho_{t}=|t\rangle\langle t| \otimes \sigma_{t}$ and the goal is to understand the evolution of $\sigma_{t}$, whose general form is

$$
\sigma_{t}=p_{t}|0\rangle\left\langle 0\left|+\bar{p}_{t}\right| 1\right\rangle\left\langle 1\left|+c_{t}\right| 0\right\rangle\left\langle 1\left|+c_{t}^{*}\right| 1\right\rangle\langle 0|,
$$

where $0 \leqslant p_{t} \leqslant 1, \bar{p}_{t}=1-p_{t}$, and $\left|c_{t}\right|^{2} \leqslant p_{t} \bar{p}_{t}$.

The action of $\hat{\mathbb{Q}}$ can change $\sigma_{t}$ in three different ways,

$$
\sigma_{t+1}=\left\{\begin{array}{l}
p_{t} \Pi_{\Omega t+\omega}+\bar{p}_{t} \Pi_{\frac{d}{2}+\omega} \text { if }|\Omega t| \leqslant K, \\
p_{t} \Pi_{\omega}+\bar{p}_{t} \Pi_{\Omega t+\omega} \text { if }\left|\Omega t-\frac{d}{2}\right| \leqslant K, \\
\hat{R}^{\omega} \sigma_{t} \hat{R}^{\omega \dagger} \text { else, }
\end{array}\right.
$$

where $\Pi_{k}=\left|\chi_{k}\right\rangle\left\langle\chi_{k}\right|$. In the first two cases, the coherences corresponding to $c_{t}$ and $c_{t}^{*}$ vanish due to the trace of the ancillary system. On the other hand, in the last case, the ancilla remains uncoupled and the oscillator evolves unitarily.

We are going to focus on how the probability $p_{t}=\left\langle 0\left|\sigma_{t}\right| 0\right\rangle$ changes in time. The formula (29) implies that $p_{t+1}$ can be derived from $p_{t}$ and $c_{t}$ via a nonhomogeneous recurrence equation with variable coefficients. It is hard to follow it analytically; therefore, we studied it numerically and the most important results are shown in Fig. 3.

In order to minimize the chance of resonances between the stimulus and the oscillator, we chose $\Omega$ and $\omega$ to be co-prime. The numerical studies show that for such a choice of frequencies, there are essentially two types of behavior. The first behavior corresponds to the situation in which there exists $t$ such that $|\Omega t| \leqslant K$ or $\left|\frac{d}{2}-\Omega t\right| \leqslant K$. In this case, the oscillator qubit gets phase locked, i.e., $p_{t}$ oscillates with frequency $\Omega$ 

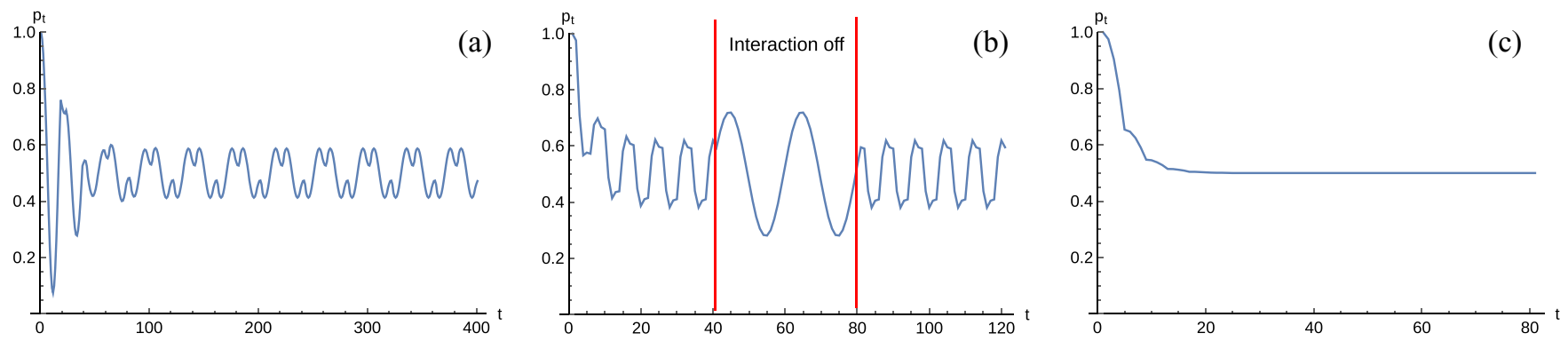

FIG. 3. Numerical simulation of $p_{t}=\left\langle 0\left|\sigma_{t}\right| 0\right\rangle$ for $d=40$. For clarity of presentation, points in the plots were connected. The singlequbit dynamics is described by Eq. (29) and the initial state is $\sigma_{0}=|0\rangle\langle 0|$. (a) $(\Omega=1, \omega=2, K=2)$ : phase locking, the qubit oscillates with the frequency $\Omega=1$, and the period of oscillations is $\frac{d}{\Omega}=40$. (b) $(\Omega=5, \omega=2, K=5)$ : the qubit is phase locked for steps $0-40$, next the interaction is turned off for steps $41-80$ and the qubit returns to its own frequency, then the interaction is turned on for steps $81-120$ and the qubit becomes phase locked again. (c) $(\Omega=5, \omega=2, K=3)$ : the qubit depolarizes to a maximally mixed state.

and the period of oscillations is $\frac{d}{\Omega}$. If the interaction between the qubit and the stimulus is turned off, the qubit returns to its own frequency, but it becomes phase locked again once the interaction is turned on-see Fig. 3(b). The second behavior corresponds to any other choice of $K$ and $\Omega$. In this case, the oscillator qubit gets depolarized and evolves towards a maximally mixed state. There is a simple explanation for the second type of behavior. In such situation, the only nontrivial application of $\hat{W}_{K}$ occurs for $\theta_{t}=0$ (and $\theta_{t}=\frac{d}{2}$, provided there exists $t$ such that $\Omega t=\frac{d}{2}$ ). For all remaining $\theta_{t}$, the oscillator qubit evolves unitarily, $\sigma_{t+1}=\hat{R}^{\omega} \sigma_{t} \hat{R}^{\omega \dagger}$. Note that for $\theta_{t}=0$ and $\theta_{t}=\frac{d}{2}$, the operator $\hat{W}_{K}$ causes an effective measurement of the oscillator qubit in the $\{|0\rangle,|1\rangle\}$ basis. Therefore, the unitary rotation about the $Y$ axis is periodically interrupted by a dephasing along the $Z$ axis, and hence the length of the qubit's Bloch vector decreases and, in the end, the qubit becomes maximally mixed.

\section{COMPARISON TO SPIN DYNAMICS}

Finally, we compare the general quantum synchronization map (13) to an open continuous dynamics of two spins. We are going to use dial states [19],

$$
|j\rangle=\frac{1}{\sqrt{2 s+1}} \sum_{s_{z}=-s}^{s} e^{i \frac{2 \pi j s_{z}}{2 s+1}}\left|s, s_{z}\right\rangle,
$$

as a natural basis to describe such system, where $j=$ $0,1, \ldots, 2 s$. These states are Fourier-transformed eigenstates of $\hat{S}_{z}$ for which the mean spin vector $\left(\left\langle\hat{S}_{x}\right\rangle,\left\langle\hat{S}_{y}\right\rangle,\left\langle\hat{S}_{z}\right\rangle\right)$ points along one of $2 s+1$ evenly distributed directions in the $x y$ plane.

Consider two spins, $s_{1}$ and $s_{2}$, that are placed in magnetic fields $B_{1} \mathbf{z}$ and $B_{2} \mathbf{z}$, respectively. The magnetic field magnitudes, $B_{1}$ and $B_{2}$, and the time of the evolution can be chosen such that the dial states are transformed into themselves and according to (11). The interaction between the spins, which would implement $\hat{G}_{K}\left(\Delta_{t}\right)$, is much harder to find. However, note that the choice of the map $G_{K}\left(\Delta_{t}\right)$ is not unique since there are many maps that lead to phase locking. The most important property of such a map is to provide a stable fixed point $\Delta^{*}$.
The general purpose of $\hat{G}_{K}\left(\Delta_{t}\right)$ is to align $s_{2}$ along $s_{1}$ using an ancillary spin $s_{3}$. In principle the spin $s_{3}$ can be used to measure in which dial states $s_{1}$ and $s_{2}$ are, and then it can be used to perform a rotation of $s_{2}$ conditioned on the measurement outcome. However, measurements in the dial basis are not easy to perform [20]. Nevertheless, one can get partial information about the dial state by measuring the $\hat{S}_{x}$ component. Therefore, let us propose the operation $\hat{G}_{K}\left(\Delta_{t}\right)$ based on the following Hamiltonian:

$$
\hat{H}_{\text {int }}=\left(\hat{S}_{x} \otimes \hat{\mathbb{1}}-\hat{\mathbb{1}} \otimes \hat{S}_{x}\right) \otimes \hat{S}_{z}+\hat{\mathbb{1}} \otimes \hat{S}_{z} \otimes \hat{S}_{x} .
$$

In the above, the tensor product is between the Hilbert spaces of $s_{1}, s_{2}$, and $s_{3}$, respectively. Intuitively, the first term in $\hat{H}_{\text {int }}$ is supposed to cause rotation of $s_{3}$ by an angle determined by the difference of $s_{1}$ 's and $s_{2}$ 's projections onto the $x$ axis. The second term is supposed to cause rotation of $s_{2}$ by the angle determined by the $s_{3}$ 's projection onto the $x$ axis. As a result, the desired transformation is a rotation of $s_{2}$ by an angle determined by the difference of $s_{1}$ 's and $s_{2}$ 's projections onto the $x$ axis. Below we apply this Hamiltonian to derive a continuous-time dynamics mimicking (13).

The spins $s_{1}, s_{2}$, and $s_{3}$ evolve according to the Hamiltonian

$$
\hat{H}_{0}=B_{1} \hat{S}_{z} \otimes \hat{\mathbb{1}} \otimes \hat{\mathbb{1}}+\hat{\mathbb{1}} \otimes B_{2} \hat{S}_{z} \otimes \hat{\mathbb{1}} .
$$

In addition, at times $t=T, 2 T, 3 T, \ldots$, spins $s_{1}$ and $s_{2}$ are coupled to $s_{3}$ via a quick impulse (kick) generated by $\exp \left(-i g \hat{H}_{\text {int }}\right)$, where $T$ is the period of time between the kicks and $g$ is the strength of the kick. We make two assumptions: (i) $s_{3}$ is prepared in the dial state $|0\rangle$ and between each kick the state of $s_{3}$ is damped back to $|0\rangle$ (irreversibility), and (ii) during the kick, the evolution generated by $H_{0}$ can be neglected. The corresponding tripartite equation of motion can be written as

$$
\dot{\rho}=-i[\hat{H}(t), \rho]+\hat{\mathcal{D}}(\rho),
$$

where

$$
\hat{H}(t)=\hat{H}_{0}+g \hat{H}_{\mathrm{int}} \sum_{n=1}^{\infty} \delta(t-n T),
$$

and $\hat{\mathcal{D}}(\rho)$ describes strong damping of $s_{3}$ to the dial state $|0\rangle$. Note that (33) is the time-continuous version of (13) in the 


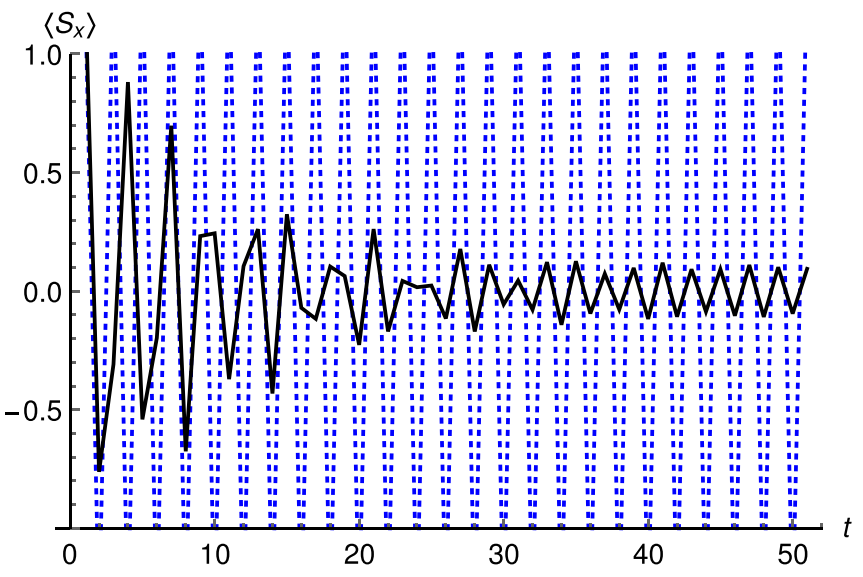

FIG. 4. The evolution of $\left\langle\hat{S}_{x}\right\rangle$ for $s_{1}$ (dashed blue lines) and $s_{2}$ (solid black lines). Both spins are initially prepared in the dial state $|0\rangle$. The plot presents a stroboscopic dynamics-only the points corresponding to times $t=k T$ are shown; however, for the clarity of presentation, these points are connected. The parameters of the evolution are $B_{1} T=2 B_{2} T=\pi$ and $g=0.5$. The transient stage before phase locking takes approximately 30 steps.

same sense as the kicked-top Hamiltonian is the continuous version of the Chirikov standard map [21].

We studied the above dynamics numerically for three spins, $s=3 / 2$. We chose $B_{1} T=2 B_{2} T=\pi$ (assuming $\hbar=$ 1 ). Such a choice guarantees that during time $T$, the spin $s_{1}$ follows a periodic transformation of dial states $|0\rangle \rightarrow|2\rangle \rightarrow$ $|0\rangle$, whereas $s_{2}$ follows $|0\rangle \rightarrow|1\rangle \rightarrow|2\rangle \rightarrow|3\rangle \rightarrow|0\rangle$. This corresponds to $\Omega=2$ and $\omega=1$. For an evolution lasting $t=100 T$, the synchronization of $s_{2}$ to $s_{1}$ can be observed for $g \geqslant 0.4$. Moreover, we assumed that all spins start in the dial state $|0\rangle$. The results for $g=0.5$ are shown in Fig. 4 . The phase locking is well visible after $t>30 T$. For such times, the period of oscillations of $\left\langle\hat{S}_{x}\right\rangle$ for $s_{2}$ is the same as the period of the corresponding oscillations for $s_{1}$. This confirms that the derived model admits synchronization.

\section{SUMMARY AND OUTLOOK}

We considered a discrete version of the PPO synchronization model and showed that the smallest classical system that can be synchronized is a single bit. Next, we quantized this model and showed that the smallest quantum system that can be synchronized is a single qubit. The quantum version allows to consider superpositions of states which, depending on the initial conditions, can either asymptotically evolve into a product of basis states or into an entangled state. We also considered a model in which a single qubit can be synchronized to a qudit. Finally, we showed that the discrete quantum synchronization model can be turned back into a continuous dynamical model of two spins that are periodically interacting with a third spin.

We focused on three particular examples of the irreversible phase-locking transformations; see Eqs. (14), (25), and (33). It is natural to investigate what possibilities other choices can offer. This might be particularly interesting in the context of quantum state engineering and quantum control. Furthermore, research on the problems discussed in this work are relevant from the point of view of complex quantum dynamics, quantum chaos, and pseudochaos. Note that phase locking in qudit systems can be considered as the emergence of some order out of pseudochaos. More precisely, if the dimension of the system and the initial frequencies of the oscillator and the stimulus are co-prime, the initial dynamics is ergodic in the sense that it visits all states in the state space. However, phase locking brakes this ergodicity and the system evolves from the $d^{2}$-dimensional Hilbert space to a highly correlated $d$-dimensional attractor subspace.

\section{ACKNOWLEDGMENTS}

The author would like to thank Andy Chia and Karol Życzkowski for helpful discussions. This work is supported by the Ministry of Science and Higher Education in Poland (science funding scheme 2016-2017 Project No. 0415/IP3/2016/74).

\section{APPENDIX A}

Here we show that for the dynamics described by Eqs. (5)(7), phase locking occurs iff $\Gamma \in E_{K}$. Let us first assume that $\Gamma \in E_{K}$. If $\Delta_{0} \in E_{K}$, then $G_{K}\left(\Delta_{0}\right)=\Delta_{0}$ and $\Delta_{1}=\Gamma$. But $\Delta_{2}=\Gamma$ as well, and therefore $\Delta^{*}=\Gamma$ and the system gets phase locked in one step. On the other hand, if $\Delta_{0} \notin$ $E_{K}$, then $G_{K}\left(\Delta_{0}\right)=0$ and $\Delta_{1}=\Delta_{0}+\Gamma$. If $\Delta_{1} \notin E_{K}$, then $\Delta_{2}=\Delta_{0}+2 \Gamma$. In general, if for all $t \leqslant \tau, \Delta_{t} \notin E_{K}$, then $\Delta_{t}=\Delta_{0}+t \Gamma$. However, there must be some $\tau(\tau<d)$ for which $\Delta_{\tau} \in E_{k}$. This is because $\Delta_{t}$ increases by $\Gamma$ and, at some point, the phase difference must fall inside $E_{K}$. Note that by assumption, $|\Gamma| \leqslant K$, and hence it is not possible to jump over $E_{K}$ while making a step $\Delta_{t} \rightarrow \Delta_{t+1}$. Therefore, $\Delta_{t}=\Delta^{*}=\Gamma$ for all $t>\tau$-the system gets phase locked in $\tau$ steps.

If $\Gamma \notin E_{K}$, then there are no fixed points. To prove it, let us assume that a fixed point $\Delta^{*}$ exists. This and (7) imply $G_{K}\left(\Delta^{*}\right)=\Gamma$, but, by definition, the values of $G_{K}\left(\Delta_{t}\right) \in E_{K}$, which contradicts the initial assumption that $\Gamma \notin E_{K}$. In this case, there is no phase locking.

\section{APPENDIX B}

To prove that $\rho_{t}$ resulting from (21) has some nonzero coherences, let us focus on the coherence between $|i+\Omega t\rangle \otimes$ $|i+\Omega t-\Gamma\rangle$ and $\left|i^{\prime}+\Omega t\right\rangle \otimes\left|i^{\prime}+\Omega t-\Gamma\right\rangle$. The crucial observation is that any initial coherence in (21) can only survive between the terms corresponding to the same initial phase difference. During phase locking, terms with different initial phase difference cause different changes of the ancilla's state and, after tracing it out, the corresponding coherence is lost. Hence, the only initial coherences from (21) that contribute to the examined final coherence stem from

$$
\left|\Delta_{0}\right\rangle=\alpha_{i} \beta_{i-\Delta_{0}}|i\rangle \otimes\left|i-\Delta_{0}\right\rangle+\alpha_{i^{\prime}} \beta_{i^{\prime}-\Delta_{0}}\left|i^{\prime}\right\rangle \otimes\left|i^{\prime}-\Delta_{0}\right\rangle,
$$

where $\Delta_{0}=0,1, \ldots, d-1$. After phase locking, these terms become

$$
\begin{aligned}
\left|\Delta_{0, t}\right\rangle= & \alpha_{i} \beta_{i-\Delta_{0}}|i+\Omega t\rangle \otimes|i+\Omega t-\Gamma\rangle \\
& +\alpha_{i^{\prime}} \beta_{i^{\prime}-\Delta_{0}}\left|i^{\prime}+\Omega t\right\rangle \otimes\left|i^{\prime}+\Omega t-\Gamma\right\rangle .
\end{aligned}
$$


The examined final coherence results from a mixture of these terms and is of the form $\alpha_{i} \alpha_{i^{\prime}}^{*} \sum_{\Delta_{0}=0}^{d-1} \beta_{i-\Delta_{0}} \beta_{i^{\prime}-\Delta_{0}}^{*}$. This value vanishes only for a set of amplitudes of measure zero and is maximal for $\left|\alpha_{i}\right|=\left|\beta_{i^{\prime}}\right|=\frac{1}{\sqrt{d}}$.
[1] S. H. Strogatz, SYNC: How Order Emerges from Chaos in the Universe, Nature and Daily Life (Hyperion, New York, 2003).

[2] T. E. Lee and H. R. Sadeghpour, Phys. Rev. Lett. 111, 234101 (2013).

[3] S. Walter, A. Nunnenkamp, and C. Bruder, Phys. Rev. Lett. 112, 094102 (2014).

[4] S. Walter, A. Nunnenkamp, and C. Bruder, Ann. Phys. 527, 131 (2015).

[5] A. Roulet and C. Bruder, Phys. Rev. Lett. 121, 053601 (2018).

[6] A. Roulet and C. Bruder, Phys. Rev. Lett. 121, 063601 (2018).

[7] M. Koppenhöfer and A. Roulet, Phys. Rev. A 99, 043804 (2019).

[8] Á. Parra-López and J. Bergli, Phys. Rev. A 101, 062104 (2020).

[9] A. Pikovsky, M. Rosenblum, and J. Kurths, Synchronization: A Universal Concept in Nonlinear Sciences, Cambridge Nonlinear Science Series (Cambridge University Press, Cambridge, 2001).

[10] S. H. Strogatz, Nonlinear Dynamics and Chaos (CRC Press, Boca Raton, FL, 2000).

[11] N. L. Holland, The dynamics of coupled oscillators, Ph.D. thesis, The Victoria University of Wellington, 2008.
[12] Y. Kuramoto, International Symposium on Mathematical Problems in Theoretical Physics, Lecture Notes in Physics, edited by H. Araki (Springer, New York, 1975), Vol. 30, p. 420.

[13] B. V. Chirikov and F. Vivaldi, Physica D 129, 223 (1999).

[14] L. Kocarev and J. Szczepanski, Phys. Rev. Lett. 93, 234101 (2004).

[15] D. Lambić, Chaos Solitons Fractals 78, 245 (2015).

[16] A. Beige, S. Bose, D. Braun, S. F. Huelga, P. L. Knight, M. B. Plenio, and V. Vedral, J. Mod. Opt. 47, 2583 (2000).

[17] A. Peres, Phys. Rev. Lett. 77, 1413 (1996).

[18] M. Horodecki, P. Horodecki, and R. Horodecki, Phys. Lett. A 223, 1 (1996).

[19] A. Peres, Quantum Theory: Concepts and Methods (Kluwer Academic Publishers, Dordrecht, 2002), p. 406.

[20] P. Kurzyński, W. Kaszub, and M. Czechlewski, J. Phys. A: Math. Theor. 43, 265303 (2010).

[21] G. Casati, B. V. Chirikov, F. M. Izrailev, and J. Ford, Stochastic Behavior of a Quantum Pendulum Under a Periodic Perturbation, Lecture Notes in Physics (Springer, Berlin, 1979), Vol. 93, p. 334 . 